\title{
Aplicativos móveis para ensino e assistência odontológica: uma revisão integrativa
}

\author{
Marcylene Arruda Braz*; Elken Gomes Rivaldo**; Flávio Renato Reis de Moura***; Raul Antônio

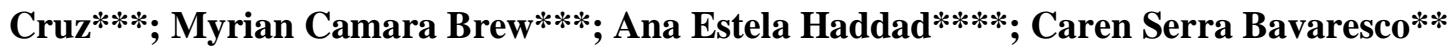 \\ * Mestranda, Programa de Pós-graduação em Odontologia, \\ ULBRA Canoas \\ ** Professora, Programa de Pós-graduação em Odontologia, \\ ULBRA Canoas \\ *** Professor(a), Curso de Odontologia, ULBRA Canoas \\ **** Professora, Programa de Pós-Graduação em Odontologia, FO- \\ USP São Paulo
}

Recebido em 09/12/2017. Aprovado em 29/07/2018.

\begin{abstract}
RESUMO
Este estudo teve como objetivo identificar o estado da arte atual e as tendências de pesquisas que utilizam aplicativos móveis na Odontologia por meio de uma revisão integrativa. Realizaram-se buscas nas bases de dados SciELO, LILACS e PubMed, utilizando os termos "mobile application”, "Dentistry" e "informatics", e no Google Acadêmico, para abarcar a literatura cinza, com os mesmos termos na língua portuguesa, sem período de tempo determinado. Os títulos e resumos de todos os artigos encontrados foram lidos por dois pesquisadores de forma independente. Foram incluídas pesquisas oriundas de dados primários e que estivessem relacionados apenas à saúde bucal, cujo meio de informação fosse smartphones, nas línguas inglesa, portuguesa e espanhola. Além disso, os artigos publicados em periódicos científicos foram analisados em relação aos itens atribuídos no checklist do STROBE Statement. Foram encontrados 911 estudos. Após a leitura de todos os títulos e resumos, foram excluídos 897, totalizando 14 estudos para leitura na íntegra. Nove artigos foram incluídos no estudo, classificados em estudos com foco profissional (6) e estudos com foco acadêmico (3). Os dados descritos nos estudos deixam claro a crescente adoção do uso dos aplicativos como recursos educacionais e assistenciais. A criação de aplicativos móveis direcionados à Odontologia é apropriada, tanto no ensino, como uma nova possibilidade de construir conhecimento, quanto para o uso profissional, já que é inegável a disseminação global do uso de dispositivos móveis.
\end{abstract}

Descritores: Aplicativos Móveis. Odontologia. Informática. 


\section{INTRODUÇÃOO}

A tecnologia móvel é uma realidade crescente na sociedade. A popularização dos celulares inteligentes, os smartphones, permitiu um novo perfil de troca, entrega e obtenção de informação entre os usuários deste serviço ${ }^{1}$. Esse uso ubíquo do smartphone, entre a maioria dos grupos demográficos, criou oportunidades para que os profissionais implementassem várias intervenções relacionadas à saúde, em tempo real, usando recursos disponíveis universalmente ${ }^{2}$. Desse modo, desenvolver soluções no formato de aplicativos móveis representa um meio eficaz de disponibilizar ferramentas e atingir o público-alvo desejado, sejam profissionais e/ou pacientes ${ }^{3}$.

$\mathrm{Na}$ Odontologia, a crescente demanda na utilização destas tecnologias tem por finalidade facilitar a prática diária do cirurgião-dentista, seja como gestor, clínico, pesquisador ou professor ${ }^{4}$. Grande parte da atividade dos profissionais de saúde consiste em processar informações: a obtenção e registro de dados sobre o paciente, as consultas aos seus colegas de profissão, a pesquisa de literatura científica específica, procedimentos diagnósticos, o planejamento e as estratégias de tratamento, a interpretação de exames ou a condução de estudos epidemiológicos ${ }^{1,5}$. Neste contexto, a utilização de dispositivos móveis pode complementar ou tornar mais ágil parte dessas atividades, otimizando o tempo de trabalho desses profissionais $\mathrm{e}$ proporcionando benefícios à população em geral.

Além disso, a literatura tem ressaltado o benefício do uso das tecnologias de informação e comunicação como ferramenta educacional em Odontologia. A forma tradicional de lecionar vem dividindo lugar com cursos de educação continuada à distância, simuladores de procedimentos clínicos, programas para comunicação em tempo real, entre outros. A importância dessas ferramentas se dá pela capacidade de permitir aos estudantes procurar e selecionar informações, aprender de forma independente e solucionar problemas ${ }^{6}$.

Nesse sentido, o objetivo desta pesquisa foi identificar, por meio de uma revisão integrativa de literatura, o estado da arte atual e as tendências de pesquisas que utilizam aplicativos móveis na Odontologia.

\section{METODOLOGIA}

Para o desenvolvimento deste estudo foi realizada uma revisão integrativa visando apreender o que existe na literatura científica nacional e internacional sobre a temática "aplicativos móveis relacionados à saúde bucal”. Sem filtros para datas, foram realizadas buscas nas bases de dados SciELO, LILACS, PubMed e Google Acadêmico, durante maio de 2017.

Para a estratégia de busca, foram utilizados os seguintes termos presentes nos Descritores em Ciências da Saúde (DeCS): "mobile application", "Dentistry" e "informatics", com o operador boleano " $A N D$ " entre cada uma das palavras. Também se pesquisou no Google Acadêmico os termos equivalentes em português ("aplicativos móveis", "Odontologia", "informática"). Foram incluídos artigos indexados, teses, dissertações e trabalhos de conclusão de curso, com delineamentos transversais e de intervenção do tipo antes e depois.

Os títulos e resumos de todos os artigos encontrados foram lidos por dois pesquisadores de forma independente. Os critérios de inclusão foram: pesquisas oriundas de dados primários e que estivessem relacionados apenas à saúde bucal, cujo meio de informação fosse smartphones, nas línguas inglesa, portuguesa e espanhola. Foram excluídos estudos da revisão e aqueles em que não foi possível identificar relação com a temática. Além disso, os artigos publicados em periódicos científicos foram 
analisados em relação aos itens de checklist do STROBE Statement.

Ao final da categorização e análise dos estudos, foi realizada a interpretação dos achados, cuja síntese apresenta-se em tabelas com o ano da publicação, local de realização da pesquisa, metodologia empregada e resultados encontrados.

\section{RESULTADOS}

A partir da busca nas bases de dados estabelecidas, obtiveram-se 911 documentos, que após remoção de estudos duplicados resultaram em 909. Não foram adicionados estudos identificados em outras fontes, ou seja, pesquisas encontradas em livros ou referências dos estudos achados nas bases de dados. Após leitura de todos os títulos e resumos, respeitando-se os critérios de inclusão e exclusão, permaneceram 14 estudos para leitura na íntegra. Para análise descritiva, foram incluídos apenas 9 estudos. A figura 1 resume as etapas da busca em um fluxograma.

Optou-se por classificar os estudos conforme o foco de seu planejamento: atuação profissional $(n=6)$ ou acadêmica $(n=3)$ (tabelas 1 e 2).

Todos os sistemas com foco acadêmico foram testados por alunos de cursos de graduação em Odontologia. No entanto, apenas um trabalho relatou o período em que esses alunos se encontravam $\left(4^{\circ}\right.$ semestre $)^{9}$.

No estudo realizado por Warmling et al. ${ }^{7}$, os alunos utilizaram o sistema para estudo dos determinantes da doença cárie em atendimentos a escolares, aplicando o questionário que busca conhecer o perfil do indivíduo e o formulário composto pelo exame físico intrabucal e percepções subjetivas do profissional. Os alunos observaram que seria mais fácil se o aplicativo fosse usado na população adulta, devido às dificuldades dos escolares ao responderem as questões como endereço e situação socioeconômica. Além disso, sugeriram que fosse incluído um odontograma e um banco de imagens no aplicativo, como apoio ao diagnóstico.

Já no estudo desenvolvido por Arus et al. ${ }^{8}$, os autores criaram um aplicativo a fim de potencializar as habilidades na interpretação da articulação temporomandibular avaliada em imagens por ressonância magnética (RM). Os alunos que utilizaram o sistema foram separados em dois grupos: teste (aprendizagem interativa) e controle (aprendizagem convencional), para posterior avaliação de seu desempenho por meio de prova objetiva, composta por questões relacionadas à posição e alterações de estruturas de articulações temporomandibulares visualizadas em imagens de RM de um banco de dados. As respostas dos alunos foram comparadas ao padrão-ouro (avaliação de três profissionais especialistas na área). $\mathrm{O}$ método de aprendizagem convencional incluiu aulas teóricas e práticas em que os alunos avaliaram, interpretaram e discutiram os mesmos exames utilizados no grupo de teste, com discussões entre estudantes e com um professor como moderador. Já no grupo teste, os usuários só interagiram com o sistema, não foram permitidas interações com um professor. Como resposta, o grupo controle (aprendizagem convencional) demonstrou melhor desempenho. No entanto, quando comparadas com as avaliações iniciais, verificou-se que os dois grupos melhoraram seus desempenhos significativamente.

No trabalho realizado por Pereira et al. ${ }^{9}$, que envolveu o desenvolvimento de um simulador virtual de realidade de anestesia local odontológica da técnica de bloqueio do nervo alveolar inferior, 23 alunos voluntários testaram o aplicativo, usando livremente o sistema, tendo os dados de utilização e evolução técnica coletados durante uma semana. Como resultado foi observado que os alunos 
relataram maior segurança para realização da técnica em pacientes após o uso do aplicativo, o que refletiu na avaliação do índice de sucesso desses alunos, que após uma semana subiu de 22,6\% para $56,5 \%$.

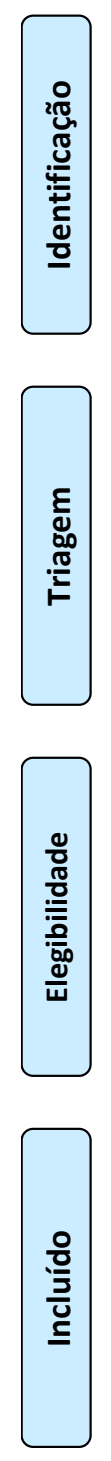

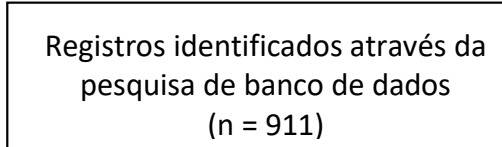

$(n=911)$
Registros adicionais identificados através de outras fontes

$$
(n=0)
$$

Registros após a remoção de duplicados

$(n=909)$

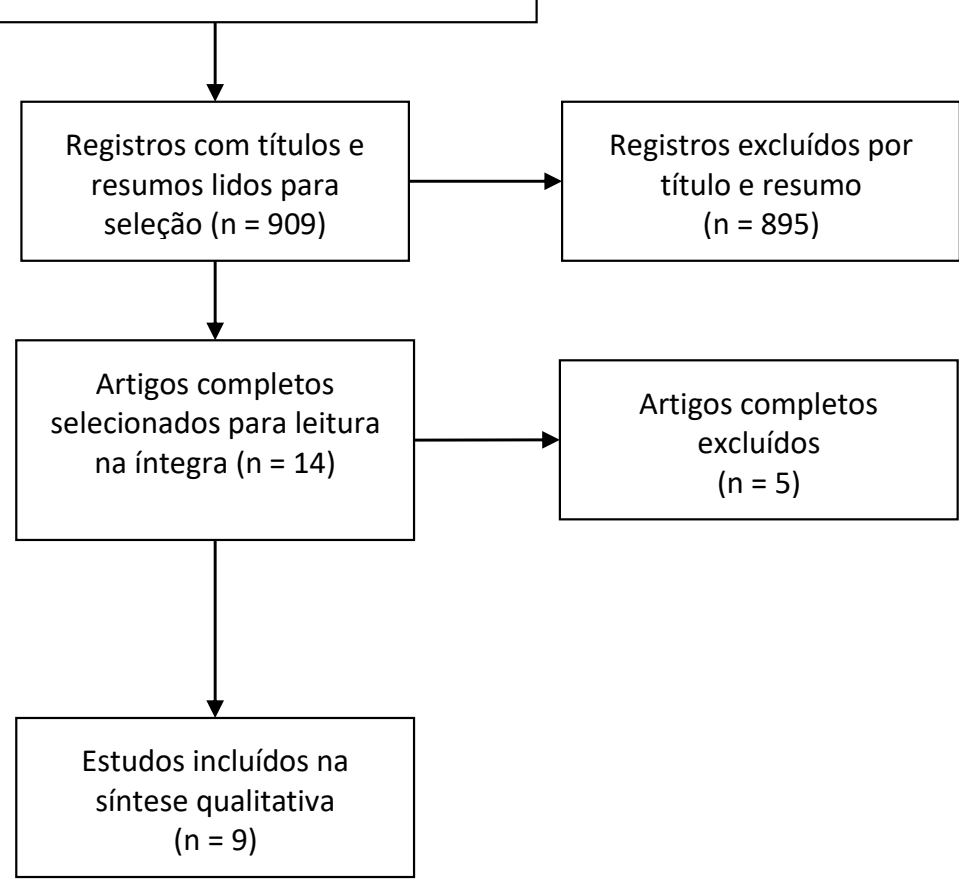

Figura 1. Fluxograma do Preferred Reporting Items for Systematic Reviews and Meta-Analyses (PRISMA) 
Quadro 1. Estudos com foco acadêmico, segundo autores/ano/país, metodologia e resultados

\begin{tabular}{|c|c|c|}
\hline \multicolumn{3}{|c|}{ Estudos com foco acadêmico } \\
\hline Autores/Ano/País & Metodologia & Resultados \\
\hline $\begin{array}{l}\text { Warmling et al. } 2012^{7} \\
\text { (Brasil) }\end{array}$ & $\begin{array}{l}\text { Aplicativo para ensino- } \\
\text { aprendizagem dos determinantes da } \\
\text { cárie dentária. Utilizado por alunos } \\
\text { de graduação em Odontologia nos } \\
\text { atendimentos a escolares }\end{array}$ & $\begin{array}{l}\text { Percepção dos usuários: sistema rápido e de fácil } \\
\text { utilização, o que também agradou aos } \\
\text { respondentes. No entanto, os alunos sugeriram } \\
\text { que fosse utilizado na população adulta, devido a } \\
\text { algumas dificuldades nas respostas das crianças. }\end{array}$ \\
\hline Arus et al., $2012^{8}$ (Brasil) & $\begin{array}{l}\text { Sistema de interpretação de imagens } \\
\text { de ressonância magnética da } \\
\text { articulação temporomandibular. } \\
\text { Testado por alunos de Odontologia. } \\
\text { A eficácia do uso do objeto foi } \\
\text { observada por meio de prova } \\
\text { objetiva. }\end{array}$ & $\begin{array}{l}\text { Grupo controle apresentou melhor desempenho. } \\
\text { Todavia, quando comparadas com as avaliações } \\
\text { iniciais, verificou-se que os dois grupos } \\
\text { melhoraram seus } \\
\text { significativamente. }\end{array}$ \\
\hline $\begin{array}{l}\text { Pereira e Ranali, } 2016^{9} \\
\text { (Brasil) }\end{array}$ & $\begin{array}{l}\text { Simulador de anestesia local } \\
\text { odontológica para ensino e } \\
\text { treinamento da técnica de bloqueio } \\
\text { do nervo alveolar inferior. }\end{array}$ & $\begin{array}{l}\text { Após uma semana de uso o índice médio de } \\
\text { sucesso dos alunos subiu de } 22,6 \% \text { para } 56,5 \% \\
(\mathrm{p}<0,001) \text {. }\end{array}$ \\
\hline
\end{tabular}

Nesta categoria, foram encontrados dois estudos voltados à coleta e armazenamento de dados de avaliações bucais obtidos em visitas domiciliares. $\mathrm{Na}$ pesquisa realizada por Forsell et al. ${ }^{10}$, desenvolveu-se, a partir das necessidades relatadas pelos trabalhadores de saída de campo da Oral Care AB (Suécia), um sistema móvel denominado MobilDent. Outro estudo com a mesma abordagem foi realizado por Figueiredo et $a .^{12}$, incluindo a utilização da geolocalização para auxiliar na localização dos domicílios, o que facilita a localização em posteriores visitas, reduzindo o trabalho envolvido de territorialização proporcionando desta forma maior rapidez na gestão de casos complexos.

Apenas quatro estudos relataram a avaliação da aplicabilidade dos sistemas. O estudo realizado por Farias e Pereira ${ }^{13}$ teve como objetivo analisar a discrepância de massa dentária de Bolton através da comparação entre a análise digital (bidimensional) com a análise manual (padrão-ouro) de 75 pares de modelos de gesso. No estudo desenvolvido por
Gomes et al. ${ }^{14}$, essa avaliação foi feita por meio de comparações entre os dados coletados pelo aplicativo e de exames padrões. Em ambas as comparações os aplicativos tiveram grande precisão em relação ao método padrão.

Já nas pesquisas realizadas por Stein et al. ${ }^{15}$, que criaram um sistema para troca de informações entre pacientes e dentistas em casos de urgências dentárias, e Figueiredo et al. ${ }^{12}$, que desenvolveram um aplicativo para coleta e armazenamento de dados com geolocalização, os testes de aplicabilidade consistiram em reuniões entre os pesquisadores para analisar o funcionamento dos sistemas.

A usabilidade dos aplicativos, ou seja, a facilidade com que os usuários têm de empregar as ferramentas, foi testada em três estudos. No sistema de rastreio de câncer bucal ${ }^{14}, 55$ indivíduos expostos a fatores de risco de câncer foram alocados para o teste. No aplicativo criado por Stein et al. ${ }^{15}$, a usabilidade foi testada com 20 potenciais usuários que simularam urgência odontológica, enquanto que o sistema de análise de discrepância de massa 
Tabela 2. Estudos com foco profissional, segundo autores/ano/país, metodologia e resultados

\begin{tabular}{|c|c|c|}
\hline \multicolumn{3}{|c|}{ Estudos com foco profissional } \\
\hline Autores/Ano/País & Metodologia & Resultados \\
\hline 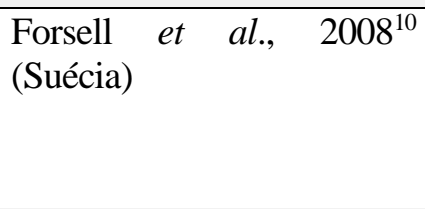 & $\begin{array}{l}\text { Recolhimento e armazenamento de dados em visitas } \\
\text { domiciliares. Projetado com base nas necessidades } \\
\text { relatadas por trabalhadores durante saída de campo. } \\
\text { Disponível na Plataforma Windows Mobile } \\
\text { (Microsoft). Não relata testes de usabilidade. }\end{array}$ & $\begin{array}{l}\text { O aplicativo foi utilizado de janeiro de } \\
2006 \text { até março de } 2008 \text { para mais de } \\
40 \text { mil avaliações bucais. Os usuários } \\
\text { relataram que o sistema é } \\
\text { autoinstrutivo. }\end{array}$ \\
\hline Souza et al., 2013 $3^{11}$ (Brasil) & $\begin{array}{l}\text { Protótipo de um aplicativo destinado aos cuidados } \\
\text { bucais de pacientes com necessidades especiais. } \\
\text { Disponível nas plataformas IOS e Android. Até o } \\
\text { momento, não foi avaliada a sua usabilidade. }\end{array}$ & $\begin{array}{l}\text { Segundo os autores, faz-se necessário } \\
\text { a escolha adequada das plataformas, } \\
\text { ressaltando a importância do trabalho } \\
\text { interdisciplinar, entre profissionais da } \\
\text { saúde e do desenvolvimento } \\
\text { tecnológico. }\end{array}$ \\
\hline $\begin{array}{l}\text { Figueiredo et al. } 2013^{12} \\
\text { (Brasil) }\end{array}$ & $\begin{array}{l}\text { Sistema de coleta e armazenamento de dados com } \\
\text { geolocalização para ser utilizado em atendimentos } \\
\text { domiciliares. Plataforma Android. Não foram } \\
\text { relatados testes de aplicabilidade com pacientes, } \\
\text { apenas reuniões interdisciplinares para discutir } \\
\text { melhorias para o sistema. }\end{array}$ & $\begin{array}{l}\text { Os autores destacam a importância de } \\
\text { cirurgiões-dentistas e profissionais da } \\
\text { informática trabalharem em conjunto. }\end{array}$ \\
\hline $\begin{array}{l}\text { Farias e Pereira, 2013 } \\
\text { (Brasil) }\end{array}$ & $\begin{array}{l}\text { Aplicativo para análise da discrepância de massa } \\
\text { dentária de Bolton. A análise digital (bidimensional) } \\
\text { do software foi comparada com a análise manual } \\
\text { (padrão-ouro), de } 75 \text { pares de modelos de gesso. O } \\
\text { sistema foi avaliado por } 30 \text { ortodontistas. } \\
\text { Mutliplataforma (Java). }\end{array}$ & $\begin{array}{l}\text { Maior especificidade do programa: } \\
\text { excluir pessoas que não possuem } \\
\text { discrepância clinicamente } \\
\text { identificada. Na análise com o } \\
\text { software, foi despendido, em média, } 6 \\
\text { minutos a menos que na análise } \\
\text { manual. Cem por cento (100\%) dos } \\
\text { ortodontistas gostaram do aplicativo e } \\
93 \% \text { o usariam. }\end{array}$ \\
\hline $\begin{array}{l}\text { Gomes et al., } 2016^{14} \\
\text { (Brasil) }\end{array}$ & $\begin{array}{l}\text { Aplicativo para rastreio de câncer bucal composto por } \\
\text { duas etapas: questionário com foco na predisposição } \\
\text { do câncer e obtenção de fotos e vídeos. Cinquenta e } \\
\text { cinco indivíduos com fatores de risco de câncer foram } \\
\text { recrutados para teste de usabilidade; os dados desses } \\
\text { pacientes, coletados pelo aplicativo e por exames } \\
\text { padrões, foram comparados. Plataforma Android. }\end{array}$ & $\begin{array}{l}\text { Quando comparado o uso do } \\
\text { aplicativo com o os exames padrões, a } \\
\text { sensibilidade média foi de } 91 \% \text {, } \\
\text { especificidade de em média } 90,5 \% \text { e } \\
\text { média de acurácia } 90,90 \% \text {. }\end{array}$ \\
\hline Stein et al., $2016^{15}$ (EUA) & $\begin{array}{l}\text { Sistema para troca de informações (fotos e relatórios) } \\
\text { entre pacientes e dentistas em casos de urgências } \\
\text { dentárias. Consistiu em } 4 \text { fases: (1) avaliação de } \\
\text { qualidade de imagens intra-orais capturadas por } \\
\text { smartphones, (2) desenvolvimento e (3) refinamento } \\
\text { do protótipo, e (4) avaliação através de testes de } \\
\text { usabilidade e satisfação com } 20 \text { potenciais usuários. } \\
\text { Plataforma Android. }\end{array}$ & $\begin{array}{l}\text { A qualidade de exibição de imagens } \\
\text { de condições bucais é satisfatória em } \\
\text { diferentes modelos de smartphones. } \\
\text { Durante o refinamento do protótipo, } \\
\text { foram encontradas algumas palavras e } \\
\text { opções de seleção ambíguas ou } \\
\text { redundantes, e, além disso, quando } \\
\text { perguntados sobre quando a dor } \\
\text { iniciou, não havia opções de respostas } \\
\text { precisas; essas questões foram } \\
\text { corrigidas. } \\
\text { Todos os participantes completaram } \\
\text { com sucesso a simulação de um relato } \\
\text { de caso em menos de } 4 \text { minutos e } \\
\text { relataram estar satisfeitos com o } \\
\text { aplicativo. }\end{array}$ \\
\hline
\end{tabular}


dentária ${ }^{10}$ foi testado por 30 ortodontistas. Todos os usuários destes sistemas relataram uma considerável facilidade em lidar com os aplicativos, sendo estes autoexplicativos.

Em relação à satisfação dos usuários, todos os estudos obtiveram um retorno positivo. Além disso, nenhum usuário relatou dificuldade em lidar com os sistemas. Em duas pesquisas, os autores ${ }^{11,12}$ salientaram fortemente a importância de projetos interdisciplinares envolvendo áreas da ciência da computação e da Odontologia, onde ambas se complementam na utilização da tecnologia na saúde.

\section{DISCUSSÃO}

Os resultados da presente revisão evidenciam importante crescimento do uso de aplicativos móveis na área da saúde e sua aplicabilidade dentro da odontologia, tanto do ponto de vista assistencial como educacional.

Conforme se depreende dos estudos relacionados ao ensino-aprendizagem incluídos, os alunos costumam apresentar atitudes positivas em relação ao uso de tecnologias como instrumentos de ensino $^{16}$. Por isso, reforça-se a questão do incentivo à utilização das Tecnologias de Informação e Comunicação como uma ferramenta pedagógica para potencializar a educação odontológica, sustentada na construção do conhecimento pelo aluno e no desenvolvimento de novas capacidades: inovação, criatividade, autonomia e comunicação ${ }^{17}$.

Está claro que o uso dessas tecnologias não substituirá a figura do docente, mas vai exigir-lhe um novo perfil, uma vez que as tecnologias vão acrescentar valor aos métodos tradicionais de ensino $^{6}$. O professor tende a se transformar no estimulador da curiosidade do aluno para que este deseje conhecer e pesquisar a informação mais relevante $^{17}$. Todavia, é necessário salientar que toda essa tecnologia agrega valor ao já consagrado e fundamental papel presencial da figura do docente ${ }^{6}$. No estudo realizado por Arus et al. ${ }^{8}$, os alunos que compuseram o grupo de aprendizagem convencional (controle), que incluiu aulas teóricas e práticas com um professor como moderador, demonstrou melhor desempenho que o grupo que só interagiu com o sistema. Por isso, o método tradicional de ministrar aulas não está obsoleto e o contato pessoal permanece como item importante na relação didático-pedagógica e precisa sempre ser encorajada e estimulada, mesmo com o crescente avanço da tecnologia ${ }^{6}$. Este fato sugere que as tecnologias sejam um recurso para enriquecer o processo de ensino-aprendizagem, aliado a presença do docente com o uso de tecnologias ${ }^{18}$. Nesse mesmo estudo, ambos os grupos melhoraram significativamente seu desempenho quando comparadas as avaliações iniciais e finais.

Uma vez que a capacidade dos smartphones para captura e transmissão de informações continua a crescer, é possível que profissionais utilizem esses dispositivos como recurso complementares nas suas decisões clínicas nos países onde a teleconsulta é permitida ${ }^{19,20}$. No estudo realizado por Stein et al.$^{15}$, o aplicativo para ser usado em urgências dentárias apresentou ótimos resultados de usabilidade. Entretanto, vale salientar que, embora sistemas como esse possam ser úteis para apoiar tratamentos remotos em Odontologia, não existe um dispositivo tecnológico que substitua totalmente o exame físico do paciente pl,22 $^{\text {. }}$.

Anteriormente, para realizar as avaliações de saúde bucal, apenas folhas manuscritas ou impressas contendo listas de endereço, prontuários e materiais de promoção de saúde, estavam disponíveis ${ }^{23}$. Hoje, os softwares racionalizaram essa rotina, diminuindo o tempo e os erros nos registros dos dados, como no uso do sistema Mobildent ${ }^{10}$, que eliminou 70 mil folhas de papel por ano, além de facilitar o aumento 
no número de pacientes atendidos em menor tempo.

O sistema operacional Android parece ser a primeira escolha, o que pode ser explicado pela grande diversidade de dispositivos de diferentes hardwares e fabricantes que rodam neste sistema, não ficando restritos a apenas uma marca do mercado de aparelhos móveis, o que promove uma maior difusão e popularização dos softwares ${ }^{24}$.

Dois estudos ${ }^{11,12}$ destacaram a importância da construção de aplicativos por profissionais da saúde aliados a profissionais específicos da área de desenvolvimento tecnológico, o que faz sentido, pois dessa maneira as áreas se complementam. Os cirurgiões-dentistas podem expor suas necessidades para agregar saúde e bem-estar aos seus pacientes, enquanto os profissionais sistematizam em forma de aplicativos essas ideias, agregando ao produto final maior qualidade no manejo do sistema e resultados clínicos $^{11}$. Muitas vezes os profissionais da saúde não são capazes de descobrir sozinhos como aplicar novas tecnologias em suas tarefas e atividades. Do mesmo modo, profissionais da ciência da computação encontram dificuldades em se situar e tomar conhecimento das necessidades e anseios da sociedade ${ }^{25}$. Portanto, a evidência é clara, há necessidade de conjugar os conhecimentos das duas áreas: saúde e informática, em grandes proporções contribuindo para a qualidade de vida da população.

Investigar a utilidade clínica dos sistemas é necessário para determinar a eficácia da implementação de um aplicativo na área da saúde ${ }^{3}$, no entanto, alguns estudos não relataram esses testes. Percebe-se a grande facilidade que os usuários possuem para manejar os aplicativos, retratando o perfil da sociedade atual, que está cada vez mais adaptada ao uso de smartphones ${ }^{1}$. Esse dado sugere a possibilidade de expandir a criação desses sistemas, com o objetivo de alcançar um maior número de usuários a fim de promover saúde.
Tendo em vista a crescente produção de conhecimento científico sobre o tema, a presente revisão pode não ter conseguido abranger toda a evidência científica disponível em relação ao uso de aplicativos móveis para a Odontologia. Além disso, a análise de estudos com delineamentos mais robustos dever ser realizada a fim de analisar o impacto a longo prazo da utilização destes recursos tecnológicos nos desfechos de saúde bucal.

\section{CONCLUSÕES}

Os estudos que fizeram parte desta revisão confirmam a crescente necessidade de criação de sistemas com foco na Odontologia e demais áreas da saúde, para assim atingir o público-alvo com mais facilidade, devido à inegável disseminação global do uso de dispositivos móveis. No ensino odontológico, esses recursos aparecem como uma nova possibilidade de construção do conhecimento, tanto pela parte dos alunos quanto dos professores, sem deixar de valorizar os métodos tradicionais já consagrados, que, como mostram os estudos, ainda não são substituíveis.

Identificar o que profissionais e alunos necessitam em termos de capacitação técnica é essencial para planejar e implementar novas tecnologias de maneira adequada, de acordo com as demandas necessárias.

\begin{abstract}
Mobile applications for dental education and assistance: an integrative review

This study aimed to identify the current state of the art and trends of research using mobile applications in Dentistry through an integrative review. SciELO, LILACS and PubMed databases, using the terms "mobile application", "Dentistry" and "informatics", and in Google Scholar, to cover the gray literature, with the same terms in the Portuguese language, without time limitation were
\end{abstract}


searched. The titles and abstracts of all articles found were read independently by two researchers. Studies based on primary data that were related only to oral health, whose medium of information was smartphones, in the English, Portuguese and Spanish languages were included. Also, articles published in scientific journals were analyzed in relation to the items assigned in the STROBE Statement checklist. There were 911 studies. After reading all the titles and abstracts, 897 were excluded, totaling 14 studies for reading in full. Nine articles were included in the study, classified in studies with a professional focus (6) and studies with an academic focus (3). The data described in the studies have shown the increasing adoption the applications use as educational and assistance resources. The creation of mobile applications directed to Dentistry is appropriate, both in teaching, as a new possibility to build knowledge, and for professional use, since the global dissemination of the use of mobile devices is undeniable.

Descriptors: Mobile application. Dentistry. Informatics.

\section{REFERENCIAS}

1. Burdette SD, Herchline TE, Oehler R: Practicing medicine in a technological age: using smartphones in clinical practice. Clin Infect Dis. 2008; 47:117-22.

2. Saleh A, Mosa M, Yoo I, Sheets L. A systematic review of healthcare applications for smartphones. BMC Med Inform Decis Mak. 2012;12:67.

3. Ozdalga E, Ozdalga A, Ahuja N. The smartphone in medicine: a review of current and potential use among physicians and students. J Med Internet Res. 2012;14(5):128.

4. Dotta EAV; Teles GHP. Sistemas aplicativos para uso odontológico. RGO. 2003;51(2):119-22.

5. Mosa AS, Yoo I, Sheets L. A Systematic Review of Healthcare Applications for Smartphones. BMC Med Inform Decis. 2012;12:67.
6. Fontanella VRC, Schardosim M, Lara MC. Tecnologias de informação e comunicação no ensino da Odontologia. Rev ABENO. 2007;7:6781.

7. Warmling AMF, Mello ALSF, Amante CJ. Tecnologias de informação e comunicação em Odontologia: desenvolvimento de um aplicativo auxiliar no ensino [tese]. Florianópolis (SC): Universidade Federal de Santa Catarina; 2012.

8. Arus NA, Duarte R, Silveira HLD, Silveira HED. Desenvolvimento e teste de um objeto digital de aprendizagem para interpretação das imagens por Ressonância Magnética da articulação temporomandibular [tese]. Porto Alegre (RS): Federal University of Rio Grande do Sul; 2012.

9. Pereira LAP, Ranali J. Criação, desenvolvimento, aplicação e validação de um simulador computadorizado de realidade virtual para o ensino e treinamento de bloqueio do nervo alveolar inferior [Dissertação]. Piracicaba (SP): Campinas State University; 2016.

10. Forsell M, Häggström M, Johansson O, Sjögren P. A personal digital assistant application (MobilDent) for dental fieldwork data collection, information management and database handling. Br Dent J. 2008;205(9):1-4.

11. Souza RC, Alves LAC, Haddad AE, Macedo MCS, Ciamponi AL. Processo de criação de um aplicativo móvel na área de odontologia para pacientes com necessidades especiais. Rev da ABENO 2013;13(2):58-61.

12. Figueiredo M, Barone D, Jardim LE. New challenges for informatics: design and implementation of a mobile system dedicated to improve oral health conditions. TISE 2013;9:70710.

13. Farias ACR, Pereira HSG. Bolton Freeware: aplicativo de avaliação de discrepância de massa dentária desenvolvido pelo Programa de Pós- 
graduação em odontologia/UFRN [Dissertação]. Natal (RN): Universidade Federal do Rio Grande do Norte; 2013.

14. Gomes MS et al. Development of a mobile application for oral cancer screening. Technol Health Care. 2016;1-9.

15. Stein CD, Xiang Xiao BS, Steven L, Titus KLS, Harry H, Thankam PT. A prototype mobile application for triaging dental emergencies. JADA. 2017;147(10):782-91.

16. Foster L, Knox K, Rung A, Matheos N. Dental Students' Attitudes Toward the Design of a Computer-Based Treatment Planning Tool J Dent Educ. 2011 75:1434-42.

17. Tangoa, R. T. As novas tecnologias de informação e comunicação no ensino superior: um estudo de caso na Faculdade de Odontologia da Universidade de São Paulo [tese]. São Paulo (SP): Universidade de São Paulo; 2006.

18. Pahinis $\mathrm{K}$ et al. A blended learning course taught to different groups of learners in a dental school: follow-up evaluation. J Dent Educ. 2008; 72 (9):1048-57.

19. Aziz S, Ziccardi V. Telemedicine using smartphones for oral and maxillofacial surgery consultation, communication, and treatment planning. J Oral Maxillofac Surg. 2009;67(11):2505-9.
20. Namakian M, Subar P, Glassman P, Quade R, Harrington M. Inperson versus "virtual" dental examination: congruence between decision making modalities. J Calif Dent Assoc. 2012;40(7):587-95.

21. Favero L, Paven L, Arreghini A. Communication through telemedicine: home teleassistance in orthodontics. Eur J Pediatr Dent. 2009;10(4):16367.

22. Anderson R. Patient expectations of emergency dental services: a qualitative interview study. $\mathrm{Br}$ Dent J. 2004;197(6):331-34.

23. Wu R C, Straus S E. Evidence for handheld electronic medical records in improving care: a systematic review. BMC Med Inform Decis Mak. 2006;6:26.

24. Miller RH, Hillman JM, Given RS. Physician's use of electronic medical records: barriers and solutions. Health Aff (Millwood). 2004;1(18):7280.

25. Correia R, Kon F, Kon R. "Borboleta: A mobile telehealth system for primary homecare". 23rd Annual ACM Symposium on Applied Computing - Track on Computer Applications in Health Care 2008: 1343-47.

Correspondência para:

Caren Serra Bavaresco

e-mail: c c_bavaresco@yahoo.com.br

Av. Farroupilha 8001

92425-900 Canoas/RS 